\title{
Optimizing response to gefitinib in the treatment of non-small-cell lung cancer
}

This article was published in the following Dove Press journal:

Pharmacogenomics and Personalized Medicine

I4 March 201।

Number of times this article has been viewed

Pietro Carotenuto'
Cristin Roma'
Anna Maria Rachiglio'
Raffaella Pasquale'
Renato Franco ${ }^{2}$
Giuseppe Antinolfi ${ }^{3}$
Francovito Piantedosi ${ }^{4}$
Alfonso Illiano5
Gerardo Botti ${ }^{2}$
Alessandro Morabito6
Nicola Normanno
Antonella De Luca7
'Pharmacogenomic Laboratory,
CROM - Centro Ricerche
Oncologiche di Mercogliano, Avellino,
Italy; ${ }^{2}$ Surgical Pathology Unit, INT
Fondazione "G. Pascale", Naples, Italy;
${ }^{3}$ Surgical Pathology Unit, Monaldi
Hospital, Naples, Italy; ${ }^{4}$ Pneumoncology
DH Unit, Monaldi Hospital, Naples,
Italy; ${ }^{5}$ Pneumoncology Unit, Monaldi
Hospital, Naples, Italy; ${ }^{6}$ Medical
Oncology, Thoracic Department, INT
Fondazione "G. Pascale", Naples, Italy;
${ }^{7}$ Cell Biology and Biotherapy Unit, INT
Fondazione "G. Pascale", Naples, Italy

Correspondence: Nicola Normanno Cell Biology and Biotherapy Unit, INT-Fondazione Pascale, Via M. Semmola, 8013 I Naples, Italy

Tel/Fax +3908I 5903826

Email nicnorm@yahoo.com

\begin{abstract}
The epidermal growth factor receptor (EGFR) is expressed in the majority of non-small-cell lung cancer (NSCLC). However, only a restricted subgroup of NSCLC patients respond to treatment with the EGFR tyrosine kinase inhibitor (EGFR TKI) gefitinib. Clinical trials have demonstrated that patients carrying activating mutations of the EGFR significantly benefit from treatment with gefitinib. In particular, mutations of the EGFR TK domain have been shown to increase the sensitivity of the EGFR to exogenous growth factors and, at the same time, to EGFR TKIs such as gefitinib. EGFR mutations are more frequent in patients with particular clinical and pathological features such as female sex, nonsmoker status, adenocarcinoma histology, and East Asian ethnicity. A close correlation was found between EGFR mutations and response to gefitinib in NSCLC patients. More importantly, randomized Phase III studies have shown the superiority of gefitinib compared with chemotherapy in EGFR mutant patients in the first-line setting. In addition, gefitinib showed a good toxicity profile with an incidence of adverse events that was significantly lower compared with chemotherapy. Therefore, gefitinib is a major breakthrough for the management of EGFR mutant NSCLC patients and represents the first step toward personalized treatment of NSCLC.
\end{abstract}

Keywords: gefitinib, EGFR, NSCLC, EGFR mutations

\section{Introduction}

Non-small-cell lung cancer (NSCLC) is the major cause of cancer death worldwide. Conventional chemotherapy offers only a modest benefit for patients with advanced NSCLC, slightly prolonging survival, but at the cost of clinically significant adverse events (AEs). Novel target-based agents, which act on specific signaling pathways involved in cell proliferation and survival, have emerged as effective agents in treating NSCLC. ${ }^{1}$

The epidermal growth factor receptor (EGFR) (HER1, ErbB-1) is a tyrosine kinase receptor belonging to the ErbB family of receptors. The EGFR possesses an extracellular ligand-binding domain, a single hydrophobic transmembrane domain that is involved in interaction among the ErbB receptors, and an intracellular domain in which the tyrosine kinase (TK) activity resides. Ligands that belong to the EGF family of growth factors are able to activate the EGFR by inducing the formation of homo- or heterodimers with other ErbB receptors, with subsequent phosphorylation of the intrinsic TK domain. ${ }^{2,3}$ In turn, ErbB receptors activate several intracellular signaling pathways, including the RAS/RAF/MAPK pathway, which plays an important role in regulating cell proliferation, migration, and differentiation, and the PI3K/AKT pathway, which is involved in cell survival. 
The EGFR is overexpressed in a variety of common solid tumors, including NSCLC. ${ }^{3}$ The observation that the EGFR is expressed in the majority of human NSCLC led to the hypothesis that activation of this receptor might play an important role in NSCLC growth and therefore might represent a suitable therapeutic target. In this regard, several anti-EGFR agents are in an advanced phase of clinical development. Two classes of EGFR inhibitors have been approved: anti-EGFR monoclonal antibodies and small-molecule inhibitors of the EGFR tyrosine kinase activity (EGFR TKIs). ${ }^{4}$ Monoclonal antibodies directed against the EGFR target the extracellular domain of the receptor to prevent ligand binding and subsequent receptor activation. EGFR TKIs compete for adenosine triphosphate (ATP) binding in the TK domain of the receptor, thus blocking EGFR autophosphorylation and downstream signaling pathways. The EGFR TKIs gefitinib and erlotinib have been approved for the treatment of NSCLC. ${ }^{3}$ In particular, erlotinib was approved as second- and third-line treatment of patients with advanced NSCLC on the basis of the results of the Phase III trial BR.21 in which a survival advantage in unselected patients treated with the drug, compared with patients treated with placebo, was demonstrated. ${ }^{5}$ Gefitinib, in the ISEL (Iressa Survival Evaluation in Lung Cancer) trial, failed to show an improvement of survival in an unselected population but showed a benefit in never-smokers and Asian patients. ${ }^{6}$ These results, as well as findings from Phase II studies of gefitinib in NSCLC, led to the hypothesis that only patients with well-defined clinicopathological and molecular features might benefit from EGFR TKIs.

In 2004, three landmark papers identified in NSCLC patients mutations in the TK domain of the EGFR that predicted response to erlotinib and gefitinib. ${ }^{7-9}$ These novel EGFR mutations rendered tumors dramatically more sensitive to the effects of EGFR TKIs compared with tumors carrying the wild-type receptor. As we will detail in the next paragraphs, the results of clinical trials have clearly demonstrated that treatment with EGFR TKIs produces an extremely high response rate and a significant improvement of survival in NSCLC patients with EGFR mutations. These data induced the European Medicines Agency (EMEA) to approve the use of gefitinib in all lines of therapy for patients carrying a mutant EGFR. As a matter of fact, this decision has established a new paradigm for the treatment of NSCLC patients and represents the first step toward personalized therapy for lung cancer patients.

\section{EGFR mutations in NSCLC}

EGFR mutations were discovered following sequencing of the EGFR gene in NSCLC patients who responded to treatment with gefitinib or erlotinib in early clinical trials. Mutations were found in the first four exons (18 through 21) of the TK domain of the EGFR (Figure 1). A number of different mutations have been described. ${ }^{10}$ However, only nine mutations have been reported with a frequency higher than $1 \%$, and approximately 160 mutations have been described only once. ${ }^{10}$ The most frequent EGFR mutations are a group of small in-frame deletions in exon 19 and a singlepoint mutation in exon 21 . The exon 19 deletions include amino acid residues around codons 746 to 750 ( $\triangle$ ELREA) and account for $45 \%-50 \%$ of all EGFR TK mutations. ${ }^{11-14}$ In exon 21, a single-point mutation that substitutes an arginine for a leucine at codon 858 (L858R) represents $40 \%-45 \%$ of all EGFR TK-activating mutations. Other less frequent EGFR-activating mutations include a glycine-719 change to serine, alanine, or cysteine (G719X) in exon 18 and in-frame duplications, insertions, or point mutations in exon 20 (Figure 1).

Kinase domain mutations of the EGFR are referred to as "activating mutations" because they lead to more persistent activation of the TK in response to exogenous growth factors compared with the wild-type receptor. ${ }^{7}$ Activating EGFR mutations involve the ATP-binding pocket in the receptor TK domain, which is the binding site for erlotinib and gefitinib. In this regard, it has been demonstrated that activating EGFR

\section{EGFR TK domain}

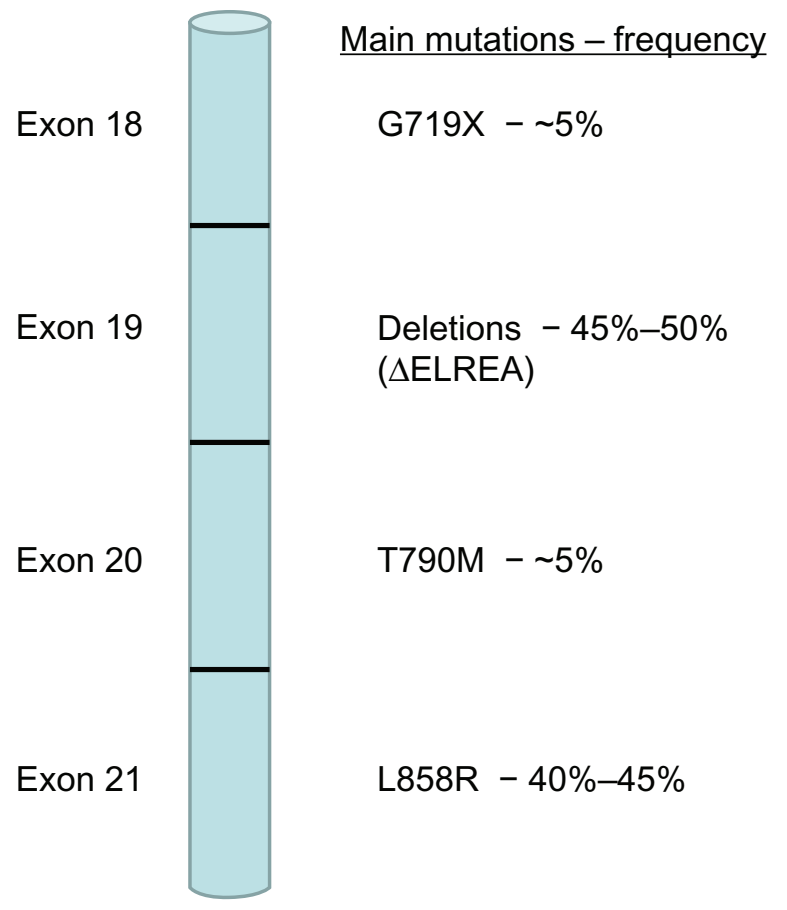

Figure I Frequency of main epidermal growth factor receptor (EGFR) mutations in non-small-cell lung cancer. 
mutations also lead to increased affinity of gefitinib and erlotinib for the mutant receptor compared with the wild-type receptor. ${ }^{9}$ As a consequence, mutant EGFR is sensitive to lower concentrations of gefitinib compared with wild-type receptor. Similar data were obtained for erlotinib. ${ }^{9}$

NSCLC carrying a mutant EGFR is highly dependent on the EGFR pathway for its growth, ie, an "oncogenic addiction" to the EGFR pathway occurs. ${ }^{12}$ In contrast, current evidence suggests that lung tumors that carry a wild-type EGFR are not highly sensitive to inhibition of this pathway. The relationship between EGFR mutations and clinical responses to EGFR TKIs has been extensively characterized in NSCLC. Overall, activating EGFR mutations are strongly associated with increased response to EGFR TKIs. ${ }^{12}$ However, it must be emphasized that the majority of available clinical data are related to the most frequent mutations, ie, the exon 19 deletions and the L858R-point mutation. Exon 18 G719X mutations are also likely associated with a response to EGFR TKIs. However, data on survival following treatment with EGFR TKIs in patients carrying these mutations are not available. In contrast, a group of mutations in exon 20 has been associated with resistance to these agents. ${ }^{12}$ These mutations are often associated with sensitizing mutations in exons 18,19 , or 21 . The most characterized resistance mutation is the T790M-point mutation in exon 20, which increases the affinity of the EGFR for ATP, thus reducing the binding of EGFR TKIs to the ATP pocket. ${ }^{15}$

An interesting feature of EGFR mutations in NSCLC is that they are strongly associated with particular clinical and pathological features. In fact, EGFR mutations are far more frequent in female patients compared with male patients $(38.7 \%$ vs $10 \%)$, in the adenocarcinoma subtype compared with other histologic types (29.4\% vs $1.8 \%)$, in nonsmokers compared with smokers or former smokers (45.8\% vs 7.1\%), and in East Asian NSCLC patients (33.4\%) compared with non-East Asian patients (5.5\%). ${ }^{2}$ These clinical, pathological, and molecular characteristics identify a specific, uncommon subtype of lung cancer. The growth of this type of tumor is strictly dependent on the activation of the EGFR pathway, which might represent the leading pathway in inducing cellular transformation in these selected patients. Indeed, expression of activating EGFR mutations (either exon 19 deletions or the L858R mutation) in the lung tissue of transgenic mice results in the development of lung tumors that histologically resemble human NSCLC carrying the EGFR mutations and that are extremely sensitive to EGFR inhibition. ${ }^{16}$

\section{Efficacy of gefitinib}

The activity of gefitinib as a single agent in pretreated advanced NSCLC patients was first evaluated in the randomized Phase II IDEAL 1 (Iressa Dose Evaluation in Advanced Lung Cancer) and IDEAL 2 trials. ${ }^{17,18}$ An objective response rate of $18 \%$ in IDEAL $1 \%$ and $10 \%$ in IDEAL 2 was observed. Median survival was 7 months, and 1-year overall survival (OS) was $27 \%-35 \%$, similar to that expected with chemotherapy. These trials led to the US Food and Drug Administration (FDA) approving gefitinib as salvage third-line therapy for NSCLC. Two randomized Phase III clinical trials (INTACT 1 [Iressa NSCLC Trials Assessing Combination Treatment] and INTACT 2) examined the efficacy of first-line treatment with a combination of platinum-based chemotherapy and gefitinib. ${ }^{19,20}$ The combined treatment of chemotherapy plus gefitinib was well tolerated. However, both INTACT studies failed to demonstrate that the addition of gefitinib to chemotherapy produces a survival benefit. A placebo-controlled randomized Phase III trial (ISEL) of gefitinib as second- or third-line treatment in chemotherapy-refractory NSCLC patients failed to show that gefitinib was effective in improving survival. ${ }^{6}$ Median survival was 5.6 months in the gefitinib group and 5.1 months in the placebo group, and 1-year OS was $27 \%$ versus $21 \%$, respectively. In June 2005, on the basis of the lack of survival benefit of the ISEL study, the FDA restricted the use of gefitinib to patients participating in a clinical trial or continuing to benefit from treatment already initiated. Four randomized Phase III trials compared gefitinib and docetaxel as second-line treatment of advanced NSCLC patients. The largest study was INTEREST (Iressa NSCLC Trial Evaluating Response and Survival Against Taxotere), which evaluated the effectiveness of gefitinib versus docetaxel in patients with locally advanced or metastatic NSCLC pretreated with platinum-based chemotherapy. ${ }^{21}$ The trial enrolled 1466 patients from 149 centers in 24 countries. The results of this study demonstrated the noninferiority of gefitinib in terms of OS: median OS was 7.6 months in the gefitinib group and 8.0 months in the docetaxel group, and 1-year survival was $32 \%$ and $34 \%$, respectively. ${ }^{21}$

Although the results of the INTEREST study propose that gefitinib might have activity in unselected NSCLC patients, different observations suggest that only patients carrying EGFR mutations are highly sensitive to gefitinib and are likely to significantly benefit from treatment with this drug. In the IDEAL 1 trial, a higher response rate was seen in Japanese patients than in a predominantly European-derived population (27.5\% vs $10.4 \%) .{ }^{17}$ In several studies in Caucasian patients, 
partial clinical responses to gefitinib have been observed most frequently in women, in nonsmokers, and in patients with adenocarcinoma. ${ }^{22,23}$ Subgroup analyses of patients in the ISEL trial identified a unique subpopulation of patients (Asian, female, never-smokers, adenocarcinoma histology) who were most likely to respond to EGFR TKIs. ${ }^{6}$ All these features are clearly related to a higher frequency of EGFR mutations in subgroups of patients with specific clinical and pathological features, as previously specified. In this regard, a subgroup analysis of the INTEREST trial showed that treatment with gefitinib in patients carrying mutant EGFR produced a significant improvement in progression-free survival (PFS) (hazard ratio [HR] $0.16 ; P=0.001$ ) and response rate (42.1\% vs $21.1 \%$ ) compared with docetaxel. ${ }^{24}$ In patients with EGFR mutations, an improvement in survival in both gefitinib and docetaxel groups (median survival 14.2 and 16.6 months, respectively) compared with EGFR wild-type patients (6.4 and 6.0 months, respectively) was also observed. The difference for survival between the two treatments in EGFR mutant patients was not statistically significant (HR 0.83, $P=0.60$ ). In contrast, in patients with wild-type EGFR, the response rate was higher in the docetaxel arm (9.8\% vs 6.6\%), whereas no significant difference was found for PFS (HR 1.24; $P=0.14$ ) and OS (HR 1.02, $P=0.91)$. In addition, a series of Phase II clinical trials has specifically selected patients with documented EGFR mutations to enrich the population of subjects who are most likely to benefit from first-line treatment with EGFR TKI therapy. ${ }^{25-29}$ These studies have uniformly demonstrated impressive response rates in the range of $50 \%-70 \%$ with excellent PFS and OS rates. These trials also exhibit notably improved treatment tolerance compared with conventional platinum-based doublet chemotherapy regimens, despite the inclusion in some studies of elderly patients with poor performance status.
The superiority of gefitinib compared with chemotherapy in patients with EGFR mutations in the first-line setting was confirmed in four randomized Phase III studies that enrolled NSCLC patients on the basis of molecular or clinical characteristics (Table 1). The randomized Phase III IPASS (Iressa Pan-Asia Study) compared gefitinib monotherapy with intravenous carboplatin (C) and paclitaxel (P) chemotherapy as first-line treatment in 1217 clinically selected chemotherapy-naïve Asian patients (never or light smokers and with adenocarcinoma histology) with advanced NSCLC. ${ }^{30}$ In this study, gefitinib produced a significantly superior PFS compared with $\mathrm{C} / \mathrm{P}$ (HR 0.74), exceeding the primary objective that was the noninferiority of gefitinib versus chemotherapy. However, the most interesting data from this study derived from the analysis of EGFR mutations that were carried in approximately one-third of the enrolled patients $(n=437)$. In this clinically selected population of patients, the mutation rate was high, with 261 of 437 available samples (60\%) harboring an EGFR mutation. In the subgroup of patients with mutant EGFR, PFS was significantly longer (HR 0.48) and the response rate was significantly higher with gefitinib compared with $\mathrm{C} / \mathrm{P}(71.2 \%$ vs $47.3 \%)$. In contrast, in patients carrying the wild-type receptor, PFS was significantly shorter (HR 2.85) and response rate was significantly lower with gefitinib (1.1\% vs $23.5 \%) .{ }^{30}$ Recently, OS data have been reported showing no difference between gefitinib and chemotherapy in the whole population (18.8 months with gefitinib vs 17.4 months with chemotherapy, HR $0.90, P=0.11$ ) and in the mutation-positive subgroup (21.6 months with gefitinib vs 21.9 months with chemotherapy, HR 1.00) (Table 1). ${ }^{31}$ However, this result might be due to the crossover of mutant patients from the chemotherapy to the gefitinib arm. In fact, after discontinuation of the assigned treatment, $39.5 \%$ of the patients in the C/P group received an EGFR TKI.

Table I Phase III clinical trials of gefitinib versus chemotherapy in non-small-cell lung cancer patients harboring epidermal growth factor receptor (EGFR) mutations

\begin{tabular}{|c|c|c|c|c|c|c|c|}
\hline \multirow[t]{2}{*}{$\begin{array}{l}\text { Study } \\
\text { (reference) }\end{array}$} & \multirow[t]{2}{*}{ Treatment } & \multirow[t]{2}{*}{$\mathbf{N}$} & \multicolumn{2}{|c|}{$\begin{array}{l}\text { Progression-free } \\
\text { survival (months) }\end{array}$} & \multirow[t]{2}{*}{$\begin{array}{l}\text { Hazard ratio } \\
(95 \% \mathrm{Cl})\end{array}$} & \multicolumn{2}{|c|}{$\begin{array}{l}\text { Overall survival } \\
\text { (months) }\end{array}$} \\
\hline & & & Gefitinib & Chemotherapy & & Gefitinib & Chemotherapy \\
\hline \multicolumn{8}{|c|}{ Subset analyses of subgroups of patients with EGFR mutations } \\
\hline IPASS $^{31}$ & $\begin{array}{l}\text { First-line gefitinib vs } \\
\text { carboplatin plus paclitaxel }\end{array}$ & 261 & 9.5 & 6.3 & 0.48 & 21.6 & 21.9 \\
\hline First-SIGNAL ${ }^{32}$ & $\begin{array}{l}\text { First-line gefitinib vs } \\
\text { gemcitabine plus cisplatin }\end{array}$ & 42 & 8.5 & 6.7 & 0.61 & 30.6 & 26.5 \\
\hline \multicolumn{8}{|c|}{ Trials recruiting patients with EGFR mutations } \\
\hline WJTOG $3405^{33}$ & $\begin{array}{l}\text { First-line gefitinib vs } \\
\text { cisplatin plus docetaxel }\end{array}$ & 172 & 9.2 & 6.3 & 0.489 & & \\
\hline $\mathrm{NEJ} 002^{34}$ & $\begin{array}{l}\text { First-line gefitinib vs } \\
\text { carboplatin plus paclitaxel }\end{array}$ & 224 & 10.8 & 5.4 & 0.30 & 30.5 & 23.6 \\
\hline
\end{tabular}


In First-SIGNAL (First-line Single Agent Iressa versus Gemcitabine and Cisplatin Trial in Never-smokers with Adenocarcinoma of the Lung), 309 Korean never-smoker patients with untreated lung adenocarcinoma were randomized to receive either Iressa at $250 \mathrm{mg}$ daily or cisplatin/ gemcitabine. The study failed to reach its primary endpoint of OS. Molecular analyses showed that the mutation rate in this study was $43.8 \%$. Importantly, in patients carrying an EGFR mutation, the response rate was $84.6 \%$ for gefitinib versus $37.5 \%$ for chemotherapy $(P=0.002)$, whereas in the mutation-negative subgroup the response rate was $29.9 \%$ for gefitinib and $51.9 \%$ for chemotherapy $(P=0.051)$. Furthermore, in the EGFR-mutant subgroup, the PFS was longer for patients receiving gefitinib compared with those having chemotherapy ( 8.5 versus 6.7 months), although such difference was not statistically significant (HR 0.613, $P=0.0849$ ). No significant difference in OS was observed between gefitinib and chemotherapy in the EGFR mutant and in the whole population of patients, presumably due to the poststudy use of EGFR TKIs in $80 \%$ of the patients in the chemotherapy arm (Table 1). ${ }^{32}$

The results of two randomized Phase III studies that enrolled Japanese, EGFR-mutant patients with advanced NSCLC have recently been reported. In the WJTOG3405 (West Japan Oncology Group) trial, 172 EGFR-mutant patients were randomly assigned to receive gefitinib $(250 \mathrm{mg}$ daily) or chemotherapy (cisplatin plus docetaxel). ${ }^{33}$ The study showed a median PFS of 9.2 months in the gefitinib group versus 6.3 months in the chemotherapy group (HR 0.489). The response rate was $62.1 \%$ and $32.2 \%$ with gefitinib and chemotherapy, respectively $(P<0.0001)$ (Table 1$)$.

The Phase III NEJ002 (North East Japan Gefitinib Study Group) trial compared gefitinib with chemotherapy with carboplatin and paclitaxel as first-line treatment in advanced NSCLC patients selected for EGFR mutation. ${ }^{34}$ The study was stopped by an independent data and safety monitoring committee after the preplanned interim analysis, because it showed a significant difference in PFS between the two treatment groups (median PFS 10.4 months for gefitinib versus 5.5 months for chemotherapy, HR 0.36). The final analysis confirmed these results, showing a median PFS of 10.8 versus 5.4 months for gefitinib and chemotherapy, respectively (HR 0.30), and a response rate significantly higher in the gefitinib group compared with in the chemotherapy group $(73.7 \%$ vs $30.7 \%, P<0.001)$. The OS did not differ significantly between the two treatment groups (median survival time 30.5 months for the gefitinib group and 23.6 months for chemotherapy). However, among 112 patients who completed chemotherapy, 106 (94.6\%) received second-line gefitinib, and $58.5 \%$ of these patients had a response (Table 1). ${ }^{34}$

\section{Safety and tolerability}

The safety profile of gefitinib was first defined in the Phase II IDEAL 1 and 2 trials. ${ }^{17,18}$ No unexpected AEs were observed at dosages of $250 \mathrm{mg} /$ day and $500 \mathrm{mg} /$ day, confirming the results obtained in Phase I trials. The most frequent grade 1/2 drug-related AEs were diarrhea and skin reactions. Grade 3/4 drug-related AEs were nausea, vomiting, and liver enzyme elevation. Although the profile of grade $1 / 2 \mathrm{AEs}$ was similar for both doses, grade $1 / 2 \mathrm{AEs}$ were more frequently reported with the gefitinib dosage of $500 \mathrm{mg} /$ day. Furthermore, in both IDEAL trials, a higher incidence of grade 3/4 drugrelated AEs was reported in patients receiving gefitinib $500 \mathrm{mg} /$ day than in those receiving the lower dose. Dose interruptions were mainly due to skin reactions, gastrointestinal disturbances, and elevated transaminases. Withdrawal due to drug-related AEs was observed in $1.9 \%$ of patients receiving gefitinib $250 \mathrm{mg}$ /day and $9.4 \%$ of patients receiving $500 \mathrm{mg} /$ day in the IDEAL 1 trial, and in $1 \%$ of patients receiving gefitinib $250 \mathrm{mg} /$ day and $4 \%$ of those receiving $500 \mathrm{mg} /$ day in the IDEAL 2 trial. ${ }^{17,18}$

Because the two doses of gefitinib $(250 \mathrm{mg} /$ day and $500 \mathrm{mg} /$ day) showed a similar response rate but toxicity was greater at $500 \mathrm{mg}$, the lower dose was chosen for further clinical studies.

Data from the large Phase III randomized trials ISEL, INTEREST, and IPASS confirmed the tolerable toxicity profile of gefitinib when administered at the dosage of $250 \mathrm{mg}$ /day (Tables 2 and 3).

In the ISEL trial, the most common AEs in the gefitinib group were grade $1 / 2$ rash and diarrhea. ${ }^{6}$ The overall frequency of grade $3 / 4$ AEs was $30 \%$ for gefitinib and $27 \%$ for placebo-treated patients (Table 3). The frequency of interstitial lung disease was similar in the two treatment groups (1\%). Few patients experienced AEs necessitating withdrawal (5\% in the gefitinib group and $2 \%$ in the placebo group), and few patients died as a result of AEs events ( $5 \%$ in the gefitinib group and $4 \%$ in the placebo group).

The majority of AEs associated with gefitinib in the INTEREST trial were mild in nature, and those most commonly reported were grade $1 / 2$ diarrhea and skin reactions. ${ }^{21}$ Common toxicity grade 3/4 AEs were reported in $9 \%$ of patients receiving gefitinib and $41 \%$ of patients receiving docetaxel (Table 3). AEs leading to drug discontinuation were reported in $4 \%$ of patients in the gefitinib group and in 
Table 2 Most frequent adverse events observed in non-small-cell lung cancer patients treated with gefitinib

\begin{tabular}{ll}
\hline Adverse event (all grade) & Frequency $^{\mathbf{a}}$ (\%) \\
\hline Rash & $37-85$ \\
Liver dysfunction & $55-70$ \\
Dry skin & $11-54$ \\
Diarrhea & $27-54$ \\
Asthenic conditions/fatigue & $10-39$ \\
Paronychia & $3-32$ \\
Stomatitis & $6-22$ \\
Nausea & $17-20$ \\
Constipation & $10-16$ \\
Alopecia & $3-11$ \\
Anorexia & $5-22$ \\
Vomiting & $13-15$ \\
Neutropenia & $5-8$ \\
Interstitial lung disease & $1-6$ \\
\hline
\end{tabular}

Notes: 'Data from IPASS, INTEREST, ISEL, NEJ002, and WJTOG3405 studies. 6 , 21,30,33,34 Range of reported frequency.

$11 \%$ of patients in the docetaxel group. AEs leading to death occurred in $1 \%$ versus $2 \%$, respectively. Lung interstitial disease was reported in $1 \%$ of patients treated with gefitinib and in $1 \%$ of patients in the docetaxel arm.

In the IPASS study, gefitinib was associated with a lower rate of grade $3 / 4$ AEs compared with $\mathrm{C} / \mathrm{P}(28.7 \%$ vs $61.0 \%)$, a lower rate of AEs leading to drug discontinuation (6.9\% vs $13.6 \%$ ), and a lower rate of dose modification due to toxic effects ( $16.1 \%$ vs $35 \%-37 \%$ for C/P).${ }^{30}$ AEs leading to death occurred in $3.8 \%$ of patients treated with gefitinib and in $2.7 \%$ of patients treated with the combination $\mathrm{C} / \mathrm{P}$. The most common treatment-related AEs were skin rash, diarrhea, and elevated liver aminotransferase levels in the gefitinib group, and neurotoxic effects, alopecia, and hematologic effects in the $\mathrm{C} / \mathrm{P}$ arm. Interstitial lung disease occurred in $2.6 \%$ of patients treated with gefitinib and in $1.4 \%$ of patients receiving chemotherapy.

Data from the WJTOG3405 trial confirmed the good profile of toxicity of gefitinib. ${ }^{33}$ The most common AEs in the gefitinib group were skin rash, liver dysfunction, dry skin, and diarrhea. AEs of grade 3 or more were infrequent with the exception of liver dysfunction. In the cisplatin plus docetaxel group, the most common AEs, which occurred in more than half of patients, were nausea, myelosuppression, fatigue, and alopecia. Interstitial lung disease was observed in $2.3 \%$ of patients in the gefitinib group.

In agreement with the other previously described trials, the NEJ002 study showed that skin rash and elevated levels of aspartate aminotransferase or alanine aminotransferase were the most common AEs in the gefitinib-treated group. ${ }^{34}$ The incidence of severe toxic effects (grade 3/4) was shown to be significantly higher in the chemotherapy group than in the gefitinib group (71.7\% vs $41.2 \%$ ) (Table 3 ). Interstitial lung disease was reported in $5.3 \%$ of patients in the gefitinib group.

Overall, these data demonstrated that gefitinib $250 \mathrm{mg} /$ day has a favorable safety profile with a low incidence of grade 3/4 AEs. The treatment was well tolerated because interruption of the administration of the drug was observed only in a low percentage of patients $(<7 \%)$. The only serious $\mathrm{AE}$ associated with the gefitinib treatment that has been reported in several studies is interstitial lung disease. However, the incidence of gefitinib-induced interstitial lung disease is higher in Japanese patients $(4 \%-6 \%)$ compared with those from other countries such as the US $(0.3 \%)$, although the reason for this geographic difference is unclear. ${ }^{35}$ Analysis of Japanese patients with NSCLC treated with gefitinib showed that positive smoking history, male gender, the coincidence of interstitial pneumonia, an older age, and a poor performance status are significantly associated with gefitinib-induced interstitial lung disease. ${ }^{36,37}$

\section{Implications for enhanced patient care, quality of life, and patient satisfaction/acceptability}

Treatment of patients with advanced NSCLC is aimed at obtaining a prolongation in survival and an improvement

Table 3 Frequency of adverse events occurring in non-small-cell lung cancer patients treated with gefitinib $250 \mathrm{mg} / \mathrm{day}$ in randomized Phase III clinical trials

\begin{tabular}{|c|c|c|c|c|c|}
\hline Study & Treatment & $\mathbf{N}$ & Grade I/4 (\%) & Grade 3/4 (\%) & Treatment discontinuation (\%) \\
\hline \multirow[t]{2}{*}{$\mathrm{ISEL}^{6}$} & Gefitinib & 1126 & 82 & 30 & 5 \\
\hline & Placebo & 562 & 71 & 27 & 2 \\
\hline \multirow[t]{2}{*}{ INTEREST ${ }^{21}$} & Gefitinib & 729 & 72 & 9 & 4 \\
\hline & Docetaxel & 715 & 82 & 41 & 11 \\
\hline \multirow[t]{2}{*}{ IPASS $^{30}$} & Gefitinib & 607 & & 28.7 & 6.9 \\
\hline & Carboplatin plus paclitaxel & 589 & & 61 & 13.6 \\
\hline \multirow[t]{2}{*}{$\mathrm{NEJ} 002^{34}$} & Gefitinib & 114 & 94.7 & 41.2 & \\
\hline & Carboplatin plus paclitaxel & 113 & 97.3 & 71.7 & \\
\hline
\end{tabular}


of disease symptoms without additive side effects. For this reason, assessment of quality of life (QoL) is considered important in clinical trials. QoL was determined in the gefitinib trials with the use of the Functional Assessment of Cancer Therapy-Lung (FACT-L) questionnaire and the Trial Outcome Index (TOI), which is the sum of the physical well-being, functional well-being, and Lung Cancer Subscale (LCS) scores of FACT-L. Symptoms were assessed with the use of the LCS score. ${ }^{38}$

The IDEAL 1 and 2 trials first showed an improvement of QoL in patients who received gefitinib. ${ }^{17,18}$ An improvement in disease-related symptoms was observed both in patients with tumor regression and in those with stable disease. In the group of patients receiving gefitinib $250 \mathrm{mg} /$ day, the rate of disease-related symptom improvement was $40.3 \%$ and $43.1 \%$ in IDEAL 1 and 2, respectively. The median time to symptom improvement was short, occurring within a few days following the start of the treatment. ${ }^{17,18}$ Importantly, in the cohort of patients receiving $250 \mathrm{mg}$ of gefitinib in the IDEAL 2 trial, a correlation was found between OS and symptom improvement. In fact, patients with symptom improvement had a median survival time of 13.6 months versus 4.6 months of patients with no improvement. The median survival of patients with symptom improvement without tumor response was 9.7 months. ${ }^{18}$

In the INTEREST study, significantly more patients had sustained and clinically relevant improvement in QoL with gefitinib than with docetaxel, as assessed by FACT-L total score $(25.1 \%$ for gefitinib vs $14.7 \%$ for docetaxel) and the FACT-L TOI (17.3\% for gefitinib vs $10.3 \%$ for docetaxel). ${ }^{21}$ Improvement in lung cancer symptoms, as assessed on the basis of the LCS scores, was observed both in patients treated with gefitinib (20.4\%) and in those treated with docetaxel (16.8\%).

An improvement of QoL in patients receiving gefitinib was observed in the IPASS study. ${ }^{30}$ Significantly more patients in the gefitinib group than in the chemotherapy group had a clinically relevant improvement in QoL, as assessed by scores on the FACT-L questionnaire (48.0\% for gefitinib vs $40.8 \%$ for chemotherapy) and scores on the TOI (46.4\% for gefitinib vs $32.8 \%$ for chemotherapy). Rates of reduction in symptoms were similar between patients who received gefitinib $(51.5 \%)$ and those who received chemotherapy (48.5\%), as determined on the basis of LCS scores. As expected, analysis of subgroups revealed that EGFR mutation-positive patients treated with gefitinib showed a greater improvement of symptoms compared with EGFR mutation-negative patients $(70.2 \%$ vs $14.6 \%$,
FACT-L score; $70.2 \%$ vs $12.4 \%$, TOI scores; $75.6 \%$ vs $20.2 \%$, LCS). Within mutation-positive patients, treatment with gefitinib produced a higher improvement of symptoms compared with chemotherapy $(70.2 \%$ vs $44.5 \%$, FACT-L score; $70.2 \%$ vs $38.3 \%$, TOI scores; $75.6 \%$ vs $53.9 \%$, LCS). In contrast, patients with no mutations of the EGFR showed an improvement of symptoms inferior with gefitinib than with chemotherapy (14.6\% vs $36.3 \%$, FACT-L score; $12.4 \%$ vs $28.8 \%$, TOI scores; $20.2 \%$ vs $47.5 \%$, LCS).

\section{Conclusion}

The decision of the EMEA to approve the use of gefitinib only for patients with advanced NSCLC who have mutations of the EGFR represents a milestone for the treatment of this disease. As a matter of fact, gefitinib is the first drug that has been approved for NSCLC on the basis of mutational analysis. The approval by EMEA was based on the evidence that treatment with gefitinib produces a significant effect on the course of the disease only in patients who carry activating mutations of the EGFR, as previously described.

Although the correlation between EGFR mutations and activity of gefitinib has been clearly demonstrated in several studies, several points still need to be addressed in order to improve the use of this drug. For example, some studies have suggested that patients carrying deletions of exon 19 have a better prognosis compared with those who have the L858R-point mutation in exon 21 when treated with an EGFR TKI. ${ }^{29}$ However, this correlation has not been confirmed in two trials in East Asian patients; therefore, it needs to be further explored in both Caucasian and East Asian subgroups. ${ }^{33,34}$ Furthermore, data on the activity of EGFR TKIs are available for the most common mutations, such as the deletions of exon 19 and the L858R mutations, whereas little information is available on the clinical outcome of patients carrying rarer mutations of exons 18 and 20. Interestingly, activity of gefitinib on the mechanisms involved in the progression of bone metastases in NSCLC patients has also been reported. ${ }^{39-42}$ However, it is not clear whether this phenomenon might be related to the presence and type of EGFR mutation.

Some patients harboring activating EGFR mutations that are associated with activity of EGFR TKIs do not respond to gefitinib or erlotinib. In this regard, it will be important to improve the methods of detection of the EGFR mutations. In fact, it has been shown that artifacts might frequently occur when mutations are investigated with direct sequencing of the polymerase chain reaction product and a low amount of tissue/DNA is available. ${ }^{43,44}$ Therefore, it will be important 
to assess whether lack of response is due to molecular mechanisms that limit the efficacy of gefitinib or to false positive results of the mutational analysis.

Finally, another important issue that needs to be addressed is the phenomenon of acquired resistance to gefitinib. Although the majority of patients with EGFR mutations respond to this drug and have a prolonged PFS, inevitably, all patients will experience progression of the disease. In this regard, it has been shown that approximately 50\% of the patients who progress following initial response to an EGFR TKI do have the T790M mutations, whereas 25\% of the patients show amplification of the gene that encodes for the MET receptor. ${ }^{3}$ In both cases, this event seems to be due to selection of tumor cells that harbor the T790M mutation or that have amplification of MET at the diagnosis. ${ }^{45-47}$ Because new agents that are able to block the activation of the T790M mutant EGFR or of MET will soon be available for clinical use, it will be important to develop techniques for the molecular monitoring of NSCLC patients who are treated with gefitinib. As a matter of fact, for most of these patients, at least two lines of therapy with biologic agents will be available in the near future.

In conclusion, the introduction of gefitinib in the therapy of EGFR-mutant NSCLC patients is a major breakthrough for the management of these patients and represents the first step toward personalized treatment for NSCLC patients.

\section{Acknowledgement}

Nicola Normanno is supported by grants from AIRC (Associazione Italiana per la Ricerca sul Cancro), the Italian Department of Health and Department of Research of the Campania Region.

\section{Disclosure}

The authors report no conflicts of interest in this work.

\section{References}

1. Janku F, Stewart DJ, Kurzrock R. Targeted therapy in non-small-cell lung cancer: is it becoming a reality? Nat Rev Clin Oncol. 2010;7(7): 401-414.

2. Normanno N, De Luca A, Bianco C, et al. Epidermal growth factor receptor (EGFR) signaling in cancer. Gene. 2006;366:2-16.

3. De Luca A, Normanno N. Predictive biomarkers to tyrosine kinase inhibitors for the epidermal growth factor receptor in non-small-cell lung cancer. Curr Drug Targets. 2010;11(7):851-864.

4. Normanno N, Bianco C, De Luca A, et al. Target-based agents against ErbB receptors and their ligands: a novel approach to cancer treatment. Endocr Relat Cancer. 2003;10(1):1-21.

5. Shepherd FA, Rodrigues Pereira J, Ciuleanu T, et al. Erlotinib in previously treated non-small-cell lung cancer. $N$ Engl J Med. 2005;353(2): 123-132.
6. Thatcher N, Chang A, Parikh P, et al. Gefitinib plus best supportive care in previously treated patients with refractory advanced nonsmall-cell lung cancer: results from a randomised, placebo-controlled, multicentre study (Iressa Survival Evaluation in Lung Cancer). Lancet. 2005;366(9496):1527-1537.

7. Lynch TJ, Bell DW, Sordella R, et al. Activating mutations in the epidermal growth factor receptor underlying responsiveness of non-small-cell lung cancer to gefitinib. $N$ Engl J Med. 2004;350(21):2129-2139.

8. Pao W, Miller V, Zakowski M, et al. EGF receptor gene mutations are common in lung cancers from "never smokers" and are associated with sensitivity of tumors to gefitinib and erlotinib. Proc Natl Acad Sci US A. 2004;101(36):13306-13311.

9. Paez JG, Janne PA, Lee JC, et al. EGFR mutations in lung cancer: correlation with clinical response to gefitinib therapy. Science. 2004; 304(5676): 1497-1500.

10. Linardou H, Dahabreh IJ, Bafaloukos D, et al. Somatic EGFR mutations and efficacy of tyrosine kinase inhibitors in NSCLC. Nat Rev Clin Oncol. 2009;6(6):352-366.

11. Sequist LV, Bell DW, Lynch TJ, Haber DA. Molecular predictors of response to epidermal growth factor receptor antagonists in non-small-cell lung cancer. J Clin Oncol. 2007;25(5):587-595.

12. Sharma SV, Bell DW, Settleman J, Haber DA. Epidermal growth factor receptor mutations in lung cancer. Nat Rev Cancer. 2007;7(3): 169-181.

13. Pao W, Chmielecki J. Rational, biologically based treatment of EGFRmutant non-small-cell lung cancer. Nat Rev Cancer. 2010;10(11): 760-774.

14. Gazdar AF. Activating and resistance mutations of EGFR in non-smallcell lung cancer: role in clinical response to EGFR tyrosine kinase inhibitors. Oncogene. 2009;28 Suppl 1:S24-S31.

15. Pao W, Miller VA, Politi KA, et al. Acquired resistance of lung adenocarcinomas to gefitinib or erlotinib is associated with a second mutation in the EGFR kinase domain. PLoS Med. 2005;2(3):e73.

16. Ji H, Li D, Chen L, et al. The impact of human EGFR kinase domain mutations on lung tumorigenesis and in vivo sensitivity to EGFR-targeted therapies. Cancer Cell. 2006;9(6):485-495.

17. Fukuoka M, Yano S, Giaccone G, et al. Multi-institutional randomized phase II trial of gefitinib for previously treated patients with advanced non-small-cell lung cancer (the IDEAL 1 trial) [corrected]. J Clin Oncol. 2003;21(12):2237-2246.

18. Kris MG, Natale RB, Herbst RS, et al. Efficacy of gefitinib, an inhibitor of the epidermal growth factor receptor tyrosine kinase, in symptomatic patients with non-small cell lung cancer: a randomized trial. JAMA. 2003;290(16):2149-2158.

19. Giaccone G, Herbst RS, Manegold C, et al. Gefitinib in combination with gemcitabine and cisplatin in advanced non-small-cell lung cancer: a phase III trial - INTACT 1. J Clin Oncol. 2004;22(5):777-784.

20. Herbst RS, Giaccone G, Schiller JH, et al. Gefitinib in combination with paclitaxel and carboplatin in advanced non-small-cell lung cancer: a phase III trial - INTACT 2. J Clin Oncol. 2004;22(5):785-794.

21. Kim ES, Hirsh V, Mok T, et al. Gefitinib versus docetaxel in previously treated non-small-cell lung cancer (INTEREST): a randomised phase III trial. Lancet. 2008;372(9652):1809-1818.

22. Jänne PA, Gurubhagavatula S, Yeap BY, et al. Outcomes of patients with advanced non-small cell lung cancer treated with gefitinib (ZD1839, "Iressa") on an expanded access study. Lung Cancer. 2004; 44(2):221

23. Miller VA, Kris MG, Shah N, et al. Bronchioloalveolar pathologic subtype and smoking history predict sensitivity to gefitinib in advanced non-small-cell lung cancer. J Clin Oncol. 2004;22(6):1103-1109.

24. Douillard JY, Shepherd FA, Hirsh V, et al. Molecular predictors of outcome with gefitinib and docetaxel in previously treated non-small-cell lung cancer: data from the randomized phase III INTEREST trial. $J$ Clin Oncol. 2010;28(5):744-752.

25. Sequist LV, Martins RG, Spigel D, et al. First-line gefitinib in patients with advanced non-small-cell lung cancer harboring somatic EGFR mutations. J Clin Oncol. 2008;26(15):2442-2449. 
26. Asahina H, Yamazaki K, Kinoshita I, et al. A phase II trial of gefitinib as first-line therapy for advanced non-small cell lung cancer with epidermal growth factor receptor mutations. Br J Cancer. 2006;95(8): 998-1004.

27. Inoue A, Suzuki T, Fukuhara T, et al. Prospective phase II study of gefitinib for chemotherapy-naive patients with advanced nonsmall-cell lung cancer with epidermal growth factor receptor gene mutations. J Clin Oncol. 2006;24(21):3340-3346.

28. Inoue A, Kobayashi K, Usui K, et al. First-line gefitinib for patients with advanced non-small-cell lung cancer harboring epidermal growth factor receptor mutations without indication for chemotherapy. J Clin Oncol. 2009;27(9):1394-1400.

29. Rosell R, Moran T, Queralt C, et al. Screening for epidermal growth factor receptor mutations in lung cancer. NEngl J Med. 2009;361(10): 958-967.

30. Mok TS, Wu YL, Thongprasert S, et al. Gefitinib or carboplatinpaclitaxel in pulmonary adenocarcinoma. NEngl J Med. 2009;361(10): 947-957.

31. Yang CH, Fukuoka M, Mok TS. Final overall survival results from a phase III, randomised, open-label, first-line study of gefitinib vs carboplatin/paclitaxel in clinically selected patients with advanced non-small cell lung cancer in Asia (IPASS). Presented at European Society of Medical Oncology Meeting; 2010 (Abstract LBA2).

32. Lee JS, Park K, Kim SW. A randomized phase III study of gefitinib (IRESSA) versus standard chemotherapy (gemcitabine plus cisplatin) as a first-line treatment for never-smokers with advanced or metastatic adenocarcinoma of the lung. Presented at: World Conference on Lung Cancer; 2009 (Abstract PRS4).

33. Mitsudomi T, Morita S, Yatabe Y, et al. Gefitinib versus cisplatin plus docetaxel in patients with non-small-cell lung cancer harbouring mutations of the epidermal growth factor receptor (WJTOG3405): an open label, randomised phase 3 trial. Lancet Oncol. 2010;11(2): 121-128.

34. Maemondo M, Inoue A, Kobayashi K, et al. Gefitinib or chemotherapy for non-small-cell lung cancer with mutated EGFR. $N$ Engl J Med. 2010;362(25):2380-2388.

35. Cohen MH, Williams GA, Sridhara R, et al. FDA drug approval summary: gefitinib (ZD1839) (Iressa) tablets. Oncologist. 2003;8(4): 303-306.
36. Ando M, Okamoto I, Yamamoto N, et al. Predictive factors for interstitial lung disease, antitumor response, and survival in non-small-cell lung cancer patients treated with gefitinib. J Clin Oncol. 2006;24(16): 2549-2556.

37. Kudoh S, Kato H, Nishiwaki Y, et al. Interstitial lung disease in Japanese patients with lung cancer: a cohort and nested case-control study. Am J Respir Crit Care Med. 2008;177(12):1348-1357.

38. Cella DF, Bonomi AE, Lloyd SR, et al. Reliability and validity of the Functional Assessment of Cancer Therapy-Lung (FACT-L) quality of life instrument. Lung Cancer. 1995;12(3):199-220.

39. Normanno N, De Luca A, Aldinucci D, et al. Gefitinib inhibits the ability of human bone marrow stromal cells to induce osteoclast differentiation: implications for the pathogenesis and treatment of bone metastasis. Endocr Relat Cancer. 2005;12(2):471-482.

40. Zampa G, Moscato M, Brannigan BW, et al. Prolonged control of bone metastases in non-small-cell lung cancer patients treated with gefitinib. Lung Cancer. 2008;60(3):452-454.

41. Pluquet E, Cadranel J, Legendre A, et al. Osteoblastic reaction in non-small cell lung carcinoma and its association to epidermal growth factor receptor tyrosine kinase inhibitors response and prolonged survival. J Thorac Oncol. 2010;5(4):491-496.

42. Garfield D, Normanno N. Osteoblastosis and activating epidermal growth factor receptor mutations: a relationship? J Thorac Oncol. 2010;5(4):415-416.

43. Marchetti A, Felicioni L, Buttitta F. Assessing EGFR mutations. N Engl J Med. 2006;354(5):526-528; author reply 526-528.

44. Marchetti A, Normanno N, Pinto C, et al. Recommendations for mutational analysis of EGFR in lung carcinoma. Pathologica. 2010;102(3): 119-126.

45. Maheswaran S, Sequist LV, Nagrath S, et al. Detection of mutations in EGFR in circulating lung-cancer cells. N Engl J Med. 2008;359(4): 366-377.

46. Godin-Heymann N, Bryant I, Rivera MN, et al. Oncogenic activity of epidermal growth factor receptor kinase mutant alleles is enhanced by the T790M drug resistance mutation. Cancer Res. 2007;67(15): 7319-7326.

47. Turke AB, Zejnullahu K, Wu YL, et al. Preexistence and clonal selection of MET amplification in EGFR mutant NSCLC. Cancer Cell. 2010;17(1):77-88
Pharmacogenomics and Personalized Medicine

\section{Publish your work in this journal}

Pharmacogenomics and Personalized Medicine is an international, peerreviewed, open access journal characterizing the influence of genotype on pharmacology leading to the development of personalized treatment programs and individualized drug selection for improved safety, efficacy and sustainability. This journal is indexed on the American Chemical

\section{Dovepress}

Society's Chemical Abstracts Service (CAS). The manuscript management system is completely online and includes a very quick and fair peer-review system, which is all easy to use. Visit http://www.dovepress. $\mathrm{com} /$ testimonials.php to read real quotes from published authors. 